\title{
A Link between Serotonin-Related Gene Polymorphisms, Amygdala Activity, and Placebo-Induced Relief from Social Anxiety
}

\author{
Tomas Furmark, ${ }^{1}$ Lieuwe Appel, ${ }^{2}$ Susanne Henningsson, ${ }^{3}$ Fredrik $\AA$ hhs, ${ }^{1}$ Vanda Faria, ${ }^{1}$ Clas Linnman, ${ }^{1}$ Anna Pissiota, ${ }^{1}$ \\ Örjan Frans, ${ }^{1}$ Massimo Bani, ${ }^{4}$ Paolo Bettica, ${ }^{4}$ Emilio Merlo Pich, ${ }^{4}$ Eva Jacobsson, ${ }^{5}$ Kurt Wahlstedt, ${ }^{5}$ Lars Oreland, ${ }^{6}$ \\ Bengt Långström, ${ }^{2,7}$ Elias Eriksson, ${ }^{3}$ and Mats Fredrikson ${ }^{1}$ \\ ${ }^{1}$ Department of Psychology, Uppsala University, SE-751 42 Uppsala, Sweden, ${ }^{2}$ Uppsala Imanet, GE Healthcare, SE-751 09 Uppsala, Sweden, ${ }^{3}$ Department of \\ Pharmacology, Göteborg University, SE-405 30 Göteborg, Sweden, ${ }^{4}$ GlaxoSmithKline, Medicine Research Centre, 37135 Verona, Italy, ${ }^{5}$ Quintiles AB Phase I \\ Services, SE-753 23 Uppsala, Sweden, ${ }^{6}$ Department of Neuroscience, Pharmacology, Uppsala University, SE-751 24 Uppsala, Sweden, and 7 Department of \\ Biochemistry and Organic Chemistry, Uppsala University, SE-751 23 Uppsala, Sweden
}

Placebo may yield beneficial effects that are indistinguishable from those of active medication, but the factors underlying proneness to respond to placebo are widely unknown. Here, we used functional neuroimaging to examine neural correlates of anxiety reduction resulting from sustained placebo treatment under randomized double-blind conditions, in patients with social anxiety disorder. Brain activity was assessed during a stressful public speaking task by means of positron emission tomography before and after an 8 week treatment period. Patients were genotyped with respect to the serotonin transporter-linked polymorphic region (5-HTTLPR) and the G-703T polymorphism in the tryptophan hydroxylase-2 (TPH2) gene promoter. Results showed that placebo response was accompanied by reduced stress-related activity in the amygdala, a brain region crucial for emotional processing. However, attenuated amygdala activity was demonstrable only in subjects who were homozygous for the long allele of the 5-HTTLPR or the G variant of the TPH2 G-703T polymorphism, and not in carriers of short or T alleles. Moreover, the TPH2 polymorphism was a significant predictor of clinical placebo response, homozygosity for the $\mathrm{G}$ allele being associated with greater improvement in anxiety symptoms. Path analysis supported that the genetic effect on symptomatic improvement with placebo is mediated by its effect on amygdala activity. Hence, our study shows, for the first time, evidence of a link between genetically controlled serotonergic modulation of amygdala activity and placebo-induced anxiety relief.

Key words: placebo; genes; phobia; serotonin; brain; functional neuroimaging

\section{Introduction}

In clinical drug development, randomized double-blind placebocontrolled trials (RCTs) have become the standard method for demonstrating superiority of an active drug over an inactive substance (i.e., a placebo). It is, however, well documented that the beneficial effects of placebo may be as large as those observed with active medication (Beecher, 1955; D. J. Stein et al., 2006). The placebo response is a phenomenon of considerable theoretical importance but also a major concern in clinical trials because it causes difficulties in establishing the efficacy of active compounds.

Functional neuroimaging techniques have been used for studying neurobiological correlates of the placebo effect in conditions such as pain, psychostimulant use, Parkinson's disease,

\footnotetext{
Received June 4, 2008; revised Sept. 29, 2008; accepted 0ct. 22, 2008

This work was supported by GlaxoSmithKline and the Swedish Research Council. We thank the staff members of Uppsala Imanet and Quintiles for providing excellent research conditions.

Correspondence should be addressed to Dr. Tomas Furmark, Department of Psychology, Uppsala University, Box 1225, SE-751 42 Uppsala, Sweden. E-mail: tomas.furmark@psyk.uu.se.

D0I:10.1523/JNEUROSCI.2534-08.2008

Copyright $\odot 2008$ Society for Neuroscience $\quad 0270-6474 / 08 / 2813066-09 \$ 15.00 / 0$
}

and depression (for review, see Benedetti et al., 2005; Faria et al., 2008). However, the majority of neuroimaging studies have investigated the acute placebo response, whereas studies using pharmaceutical RCT designs with sustained placebo treatment and statistical comparisons of placebo responders and nonresponders are mostly lacking.

The aim of the present study was to examine the ability of stress-induced brain activity and serotonin-related genes to predict the response to sustained placebo treatment in patients with social anxiety disorder (SAD). This is a common and disabling disorder characterized by fear of negative evaluation from others (American Psychiatric Association, 1994). Several brain imaging studies report enhanced responsiveness of the amygdala in SAD patients compared with controls during anxiety provocation and other aversive conditions (Tillfors et al., 2001, 2002; Stein et al., 2002; Lorberbaum et al., 2004; Phan et al., 2006) and attenuated amygdala responsiveness after successful treatment (Furmark et al., 2002, 2005). Pretreatment activity levels in the amygdala may also predict decreases in anxiety (Whalen et al., 2008). Because neuroimaging studies suggest that placebo acts on essentially the same neural pathways as those influenced by active treatment 
(Benedetti et al., 2005; Faria et al., 2008), an association between placebo responsivity and attenuated amygdala excitability could be expected in SAD.

Accumulating evidence suggests that amygdala reactivity is modulated by serotonin-related genes. Both in healthy volunteers (Hariri and Holmes, 2006) and individuals with SAD (Furmark et al., 2004), carriers of the short (s) allele of the serotonin transporter gene-linked polymorphic region (5-HTTLPR) show exaggerated amygdala responsivity to aversive stimuli compared with those who are homozygous for the long (l) allele. Exaggerated amygdala activation in response to emotional facial stimuli has also been reported in carriers of $\mathrm{T}$ alleles, compared with $\mathrm{G}$ allele homozygotes, of the G-703T (rs4570625) single-nucleotide polymorphism in the tryptophan hydroxylase-2 (TPH2) gene (Brown et al., 2005; Canli et al., 2005).

Prompted by these findings, we investigated the association between the 5-HTTLPR and TPH2 G-703T polymorphisms, stress-related amygdala activity, and placebo-induced anxiety relief in patients with SAD. Functional neuroimaging data were extracted from two RCTs in which patients were genotyped and treated with placebo for 8 weeks under double-blind conditions. We hypothesized that placebo responders would show greater attenuation of amygdala activity compared with nonresponders and that the amygdala attenuation would be compromised in carriers of $\mathrm{s}$ and $\mathrm{T}$ alleles.

\section{Materials and Methods}

\section{Study population and recruitment}

Twenty-five patients ( 9 males/16 females; age, mean \pm SD, $35.6 \pm 10.7$ years; range, 22-55) diagnosed with SAD were included in the analyses. Patients were taken from two previously unpublished RCTs that evaluated changes in regional cerebral blood flow (rCBF) after $56 \mathrm{~d}$ of pharmacological treatment by means of positron emission tomography (PET). Both RCTs were designed similarly to a previous study (Furmark et al., 2005). The clinical PET trials were performed in collaboration with Uppsala Imanet GE Healthcare, Quintiles AB Uppsala, and GlaxoSmithKline during 2003-2005, and included a total of 108 patients with SAD. There were three treatment arms in the first study and six arms in the second. Thirteen of 36 patients in the first study (cohort I) and 12 of 72 patients in the second (cohort II) were randomized to a placebo arm (see Fig. 1). Only the pooled placebo data are included herein, whereas additional data on psychoactive drug treatment will be reported separately.

Participants were recruited through newspaper advertising. After a short initial telephone interview, persons were asked to fill out a battery of social anxiety questionnaires, to be returned by mail. Face-to-face structured clinical diagnostic interviews (SCID) (First et al., 1998) were thereafter administered by a clinical psychologist. In addition, a psychiatrist (K.W.) administered the MINI interview (Sheehan et al., 1998) to exclude other serious psychiatric disorders than comorbid anxiety conditions. Medical examinations were also performed.

Main criteria for exclusion were as follows: treatment of social anxiety in the past 6 months, current serious or dominant psychiatric disorder other than SAD (e.g., psychosis, major depressive or bipolar disorder), chronic use of prescribed medication, abuse of alcohol/narcotics, pregnancy, menopause, left-handedness, previous PET examination, and any somatic/neurologic disorder that could be expected to influence the outcome of the study.

All participants met the Diagnostic and Statistical Manual of Mental Disorders, Fourth Edition (American Psychiatric Association, 1994) criteria for SAD and confessed to marked public speaking anxiety. Eighteen $(72 \%)$ of the patients receiving placebo were diagnosed with the generalized subtype. For the remaining seven patients, it could not be established with confidence that their fears encompassed most social situations, and, accordingly, they were classified as having the nongeneralized subtype (American Psychiatric Association, 1994). Eight patients (33\%) qualified for at least one comorbid anxiety diagnosis (specific phobia, $n=5$; generalized anxiety disorder, $n=2$; panic disorder, $n=1$; mild posttraumatic stress disorder, $n=1)$. SAD was, however, the main diagnosis for all participants.

Approvals were obtained from the Uppsala University Medical Faculty Ethical Review Board, the Uppsala University Isotope Committee, and the Swedish Medical Products Agency. A written informed consent was obtained from all participants after the nature and consequences of the study were explained.

\section{Treatment procedure}

GlaxoSmithKline supplied daily doses of study drugs and matching placebo for the two consecutive PET studies. Both studies used a fixed dosing schedule and were performed under double-blind conditions during an 8 week period. For all participants, the first dose was given immediately after the first PET examination and the final dose was administered 2-4 h before the final PET assessment after $56 \mathrm{~d}$ (posttreatment). Subjects did not receive any other form of treatment during the study period. Patients visited the clinic biweekly for assessments of compliance and side effects and to receive new supplies of medication. Vital signs (heart rate, blood pressure) were checked, laboratory safety tests (hematology, biochemistry, and urine analysis) were performed, and anxiety scales were administered. Pregnancy tests and electrocardiography were performed twice. Screening for alcohol and nonallowed drugs were performed at a randomly selected visit. After study completion, patients were offered additional psychiatric consultation and pharmacotherapy.

\section{PET assessments}

PET investigations were performed at Uppsala Imanet, GE Healthcare. The procedure for $\mathrm{rCBF}$ assessments, using $\left[\mathrm{H}_{2}{ }^{15} \mathrm{O}\right] \mathrm{PET}$, closely followed the protocol described by Furmark et al. (2005). Before and after chronic placebo administration, all participants were scanned during an anxiogenic public speaking task. Subjects were scanned using a 32 ring ECAT EXACT HR + scanner (Thermo Fisher Scientific/CTI), which enables acquisition of 63 contiguous planes of data with a distance of 2.46 $\mathrm{mm}$ resulting in a total axial field of view of $155 \mathrm{~mm}$.

Subjects were positioned in the scanner with the head gently fixated and a venous catheter for tracer injections was inserted. Patients were instructed to prepare a $2.5 \mathrm{~min}$ speech about a vacation or travel experience $\sim 20$ min before the initial emission scan. A 10 min transmission scan was performed using three retractable ${ }^{68} \mathrm{Ge}$ rotating line sources. The ${ }^{15} \mathrm{O}$-water tracer, $\sim 10 \mathrm{MBq} / \mathrm{kg}$ body weight, was thereafter injected intravenously. The emission scan started automatically in threedimensional mode when the bolus reached the brain (50,000 counts/s), and consisted of three $30 \mathrm{~s}$ frames.

Immediately after tracer injection, patients were asked to start speaking and continue until they received instructions to stop. The speech was performed in presence of a silently observing audience of six to eight persons. Patients were instructed to observe the audience. The speech was recorded from close distance with a portable video camera to increase observational anxiety. Directly after the speech, fear ratings were obtained (i.e., subjects rated how frightened they felt during scans).

Emission scans were reconstructed with a filter back projection using an $8 \mathrm{~mm}$ Hanning filter, resulting in a spatial resolution of $\sim 5 \mathrm{~mm}$ in the field of view. Data were corrected for photon attenuation, decay, scattered radiation, and random coincidences. After reconstruction, a summation image of the three frames was made to obtain a better statistical reference for realignment and subsequent analyses.

Participants fasted $3 \mathrm{~h}$, and refrained from tobacco, alcohol, and caffeine $12 \mathrm{~h}$, before PET investigations. The PET procedure was the same at posttreatment day 56, except that the speech topic was changed (i.e., this time subjects spoke about a present or previous occupation).

\section{Clinical outcome measures}

Response rate was determined by the Clinical Global Impression improvement item (CGI-I) (Zaider et al., 2003) administered biweekly by a psychiatrist (K.W.). Patients having a score of 1 or 2 (very much or much improved) on the CGI-I at posttest were classified as responders, whereas those having scores of 3 (minimally improved) or higher were considered to be nonresponders. Additional changes in the social anxiety symptom profile were evaluated by the clinician administered Liebowitz Social 
Anxiety Scale (LSAS) (Liebowitz, 1987) as well as self-report instruments, the Social Phobia Scale (SPS) and the Social Interaction Anxiety Scale (SIAS) (Mattick and Clarke, 1998). Changes in subjective fear ratings from pretreatment to posttreatment were evaluated using a $0-100$ (minimum to maximum) visual analog scale administered after each public speaking challenge. LSAS data were collected biweekly, and other measures were collected at pretreatment and posttreatment only by independent and blinded assessors. Clinical outcome was assessed before the study had been unblinded.

\section{Genotyping}

Blood samples for genotyping were available from 24 of the 25 subjects. To avoid mass comparisons, analyses were focused on the 5-HTTLPR and TPH2 G-703T polymorphisms only. Genomic DNA was extracted from plasma samples using a Qiamp DNA extraction kit (QIAGEN). Amplification was made by means of PCR on a PerkinElmer 9700 thermal cycler.

The primer sequences used for the 5-HTTLPR, reported by Gelernter et al. (1997), were 5' -ATGCCAGCACCTAACCCCTAATGT-3' (forward primer) and $5^{\prime}$-GGACCGCAAGGTGGGCGGGA-3' (reverse primer), resulting in a 419-bp-long PCR product for the 16-repeat-allele (long), and a 375-bp-long PCR product for the 14-repeat-allele (short). The $15 \mu$ l reaction mixture contained $50 \mathrm{ng}$ of genomic DNA, $1.5 \mathrm{~mm}$ $\mathrm{MgCl}_{2}, 0.3 \mu \mathrm{M}$ of each primer, $300 \mu \mathrm{M}$ dNTPs, and $1 \mathrm{U}$ of HotStarTaq polymerase from QIAGEN. The temperature profile consisted of an initial denaturation at $95^{\circ} \mathrm{C}$ for $15 \mathrm{~min}$, followed by 45 cycles of $30 \mathrm{~s}$ at $95^{\circ} \mathrm{C}$, $30 \mathrm{~s}$ at $66^{\circ} \mathrm{C}$, and $60 \mathrm{~s}$ at $72^{\circ} \mathrm{C}$, followed by final incubation for $7 \mathrm{~min}$ at $72^{\circ} \mathrm{C}$. PCR products were separated on a $3 \%$ agarose gel supplemented with ethidium bromide and visualized by ultraviolet transillumination.

The primer sequences used for the TPH2 G-703T (rs4570625) polymorphism were 5' -TGTGGCTAAATTGAACCCTTACCT-3' (forward primer) and 5' ${ }^{\prime}$-TGTGCTCCCGAACACTAGATCTTA-3' (reverse primer). The $20 \mu \mathrm{l}$ reaction mixture contained $50 \mathrm{ng}$ of genomic DNA, $1.5 \mathrm{mM} \mathrm{MgCl}_{2}, 0.15 \mu \mathrm{M}$ of each primer, $200 \mu \mathrm{M}$ dNTPs, and $1 \mathrm{U}$ of HotStarTaq polymerase from QIAGEN. The temperature profile consisted of initial denaturation at $95^{\circ} \mathrm{C}$ for $15 \mathrm{~min}$, followed by 45 cycles of $15 \mathrm{~s}$ at $95^{\circ} \mathrm{C}, 30 \mathrm{~s}$ at $62^{\circ} \mathrm{C}$, and $30 \mathrm{~s}$ at $72^{\circ} \mathrm{C}$, followed by final incubation for $7 \mathrm{~min}$ at $72^{\circ} \mathrm{C}$. Genotyping of the TPH2 polymorphism was performed by a Pyrosequencer PSQ 96MA and the PSQ 96 SNP Reagent kit (Pyrosequencing AB). To identify the polymorphism, $15 \mathrm{pmol}$ of the sequencing primer $5^{\prime}$-gctttttctgacttgacat-3' was used.

Neither the genotype distribution for 5-HTTLPR nor that for the TPH2 polymorphism differed significantly from Hardy-Weinberg equilibrium ( $p=0.75$ for 5-HTTLPR and $p=0.21$ for TPH2).

\section{Statistical analyses}

PET data. PET images were realigned to correct for different positions between scans (pretreatment vs posttreatment) and normalized to the stereotactic template of the Montreal Neurological Institute (MNI), using SPM2 (Wellcome Department of Cognitive Neurology, London, UK). Images were then smoothed using a $12 \mathrm{~mm}$ Gaussian kernel and scaled to give all scans the same global signal. PET data were statistically evaluated using within- and between-group comparisons defined in SPM2 with rCBF data fitted to the general linear model (Friston et al., 1995). Differential treatment effects between groups were evaluated by group by time interactions in the form of double subtractions, for example, $\left[\right.$ responders $_{\text {pre }}-$ responders $\left._{\text {post }}\right]-$ nonresponders $_{\text {pre }}-$ nonresponders $\left._{\text {post }}\right]$. Contrasts generated $t$ maps, subsequently converted to $z$ scores for interpretation. Brain locations are described as $x y z$ coordinates in the Talairach space, obtained by affine transformation of the MNI coordinates in SPM2 (www.mrc-cbu.cam.ac.uk/Imaging/mnispace.html).

Effects on rCBF were evaluated at the voxel level ( 1 voxel $=2 \times 2 \times 2$ $\mathrm{mm})$ by examining statistically significant changes $(p<0.05)$ corrected family-wise for multiple comparisons and with a minimum extension of 10 voxels. Small-volume correction was used for region-of-interest (ROI) analyses (i.e., for the amygdala and opioid network described below). In addition, exploratory whole-brain analyses were performed. Anatomical localization was guided by the Talairach atlas (Talairach and Tournoux, 1988), the Talairach Daemon (Lancaster et al., 2000), and the detailed medial temporal lobe atlas of Mai et al. (2004).
In line with our a priori hypothesis, primary analyses were focused on the amygdala. The amygdala volume was defined by the WFU PickAtlas (http://www.fmri.wfubmc.edu/), an automated method to generate region of interest masks (Maldjian et al., 2003) implemented within SPM2. Mean voxel values from the whole amygdala volume were extracted for all individual using MarsBaR (http://marsbar.sourceforge.net) and treatment effects were visualized as percentage change from pretreatment to posttreatment.

We also performed a directed search for altered $\mathrm{rCBF}$ in the opioid network previously implicated in placebo responsivity in pain and negative affect (Petrovic et al., 2002, 2005; Wager et al., 2004). This search encompassed four ROIs: the anterior cingulate cortex, orbitofrontal cortex, lateral prefrontal cortex (area 9, 10), and the brainstem, defined by the method described above (Maldjian et al., 2003). Covariations between these ROIs and the amygdala were evaluated in SPM2, using amygdala mean voxel values as the covariate of interest.

Clinical outcome and gene data. Clinical data were scanned for violations of normality and heterogeneity of variance, and between-group differences at baseline were evaluated by unpaired $t$ tests. Differential outcome at posttreatment for CGI responders versus nonresponders were evaluated using repeated measurement ANOVA for the LSAS, and analyses of covariance (ANCOVAs) with the pretest value as covariate in the statistical model for the SPS, SIAS, and fear measures.

Consistent with previous studies (Hariri et al., 2002), the 5-HTTLPR genotype was dichotomized such that subjects carrying at least one short allele (s group) were compared with subjects who were homozygous for the long allele (ll group). With regard to the TPH2 G-703T polymorphism, homozygotes for $\mathrm{G}$ allele (GG group) were compared with T allele carriers (T group) (Brown et al., 2005; Canli et al., 2005). To investigate the predictive power of baseline neural activity and the serotonin-related polymorphisms, a logistic regression analysis was performed with the CGI response (responder/nonresponder) on day 56 as dependent variable and 5-HTTLPR (11/s), TPH2 (GG/T), as well as left and right amygdala $\mathrm{rCBF}$ (mean voxel values day 0 ) as the independent variables. Shared variance between polymorphisms and amygdala change scores were calculated using ordinary least-squares regression.

Path analysis was performed to test the directionality of genetic and amygdala influences on clinical improvement (CGI-I day 56). The left and right amygdala were treated as a latent variable. Genotype was treated as independent variable, and errors were assumed to be uncorrelated. To avoid overfitting, models with more than two paths were not evaluated. The Bayesian information criterion (BIC) was used to evaluate model fit, the best fitting model having the lowest BIC value (Raftery, 1993). Moreover, the best fitting model should fulfill the criteria of a $\chi^{2}$ statistic corresponding to $p>0.05$ and a standardized root mean square residual value of $<0.08$.

Statistical analyses were performed using SPSS 12.1 (SPSS) and Mplus5 (Muthen and Muthen, 2008).

\section{Results \\ Clinical placebo response}

After unblinding, it was revealed that 25 patients, 13 in the first study and 12 in the second, hereafter referred to as cohorts I and II, had been randomized to a placebo arm (Fig. 1). The psychiatric assessment, based on the CGI-I (Zaider et al., 2003), showed that 10 patients had responded to placebo (40\%) and 15 had not (60\%) (Fig. 2A) (descriptive characteristics in Table 1).

Because of a different number of treatment arms, the likelihood of receiving placebo was 13 of $36(36 \%)$ in cohort I and 12 of $72(17 \%)$ in cohort II. However, this did not affect trial outcome differentially because there were five responders in both cohorts (Fisher's exact, $p>0.99$ ). Also, cohorts I and II did not differ with regard to baseline (day 0 ) scores on clinical outcome measures $(0.04<t<1.15 ; \mathrm{df}=23 ; p>0.26)$, age $(t=1.12 ; \mathrm{df}=$ $23 ; p>0.27$ ), sex distribution (Fisher's exact, $p>0.41$ ), and whole-brain or amygdala rCBF. Hence, to increase the statistical power, the two cohorts were merged in the subsequent analyses. 


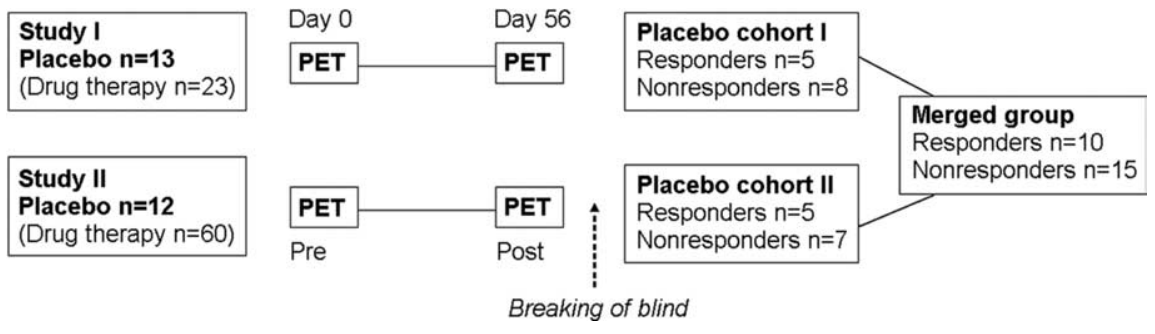

Figure 1. Study design. Patients with social anxiety disorder $(N=108)$ were randomized to 8 weeks of drug treatment or placebo under double-blind conditions in two separate studies. All participants underwent PET scanning during an anxietyprovoking public speaking task before and after treatment. Statistical analyses were based on pooled data from the two placebo cohorts.

A

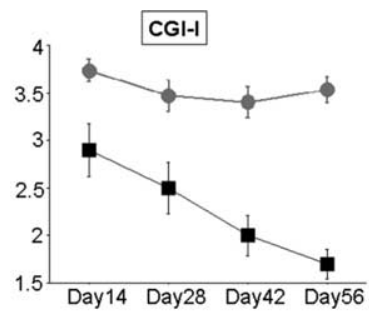

SPS
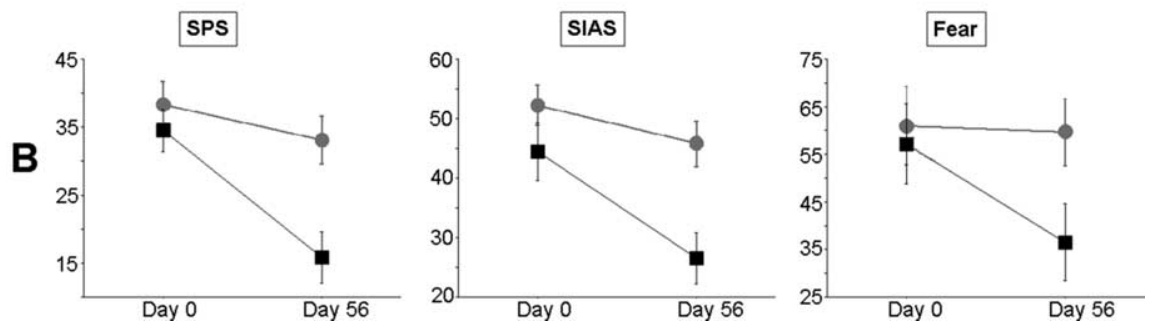

Figure 2. Mean scores ( $\pm \mathrm{SE}$ ) of clinical outcome measures for placebo responders and nonresponders. $\boldsymbol{A}$, Clinician administered outcome measures: patients having a posttreatment (day 56) score of 1-2 on the CGI-I were classified as responders ( $n=$ $10)$, and remaining patients (CGI-I $\geq 3$ ) as nonresponders ( $n=15)$. The two groups did not differ initially, but responders scored significantly lower on the LSAS at posttreatment. $\boldsymbol{B}$, Social anxiety self-report instruments: After treatment, responders and nonresponders were significantly differentiated on the SPS, SIAS, and fear ratings during the scanned public speaking task.

Patients defined as placebo responders and nonresponders by means of the CGI-I exhibited significant differences on all clinical outcome measures at posttreatment. On the LSAS (Liebowitz, 1987), a repeated measurement ANOVA yielded a significant main effect of time $\left(F=22.97\right.$; $\mathrm{df}=4,92 ; p<0.0001$; partial $\eta^{2}$ $=0.50)$ and a significant group by time interaction $(F=7.51$; df $=4,92 ; p<0.0001$; partial $\eta^{2}=0.25$ ) driven by continuous improvement in the responder group only (Fig. $2 A$ ). On social anxiety self-report instruments, ANCOVAs yielded significant effects of the group (responders/nonresponders) factor after adjusting for pretest values (SPS: $F=10.53, \mathrm{df}=1,22, p=0.004$, partial $\eta^{2}=0.32$; SIAS: $F=9.53, \mathrm{df}=1,22, p=0.005$, partial $\eta^{2}$ $=0.30$ ). Adjusted mean scores indicated lower anxiety levels (i.e., greater improvement) in responders. The same was true for subjective fear ratings during the public speech $(F=4.45$; $\mathrm{df}=1,22$; $p=0.047$; partial $\eta^{2}=0.17$ ) (Fig. $2 B$ ).

\section{PET data: amygdala}

Placebo responders versus nonresponders

The rCBF response to public speaking decreased significantly more in placebo responders relative to nonresponders in the left amygdala $\left[\left(\begin{array}{lll}x & y & z\end{array}\right)=(-12-3-13) ; z=2.64 ; p=0.048 ; 24\right.$ voxels; Cohen's $d=1.21$ ] (Fig. 3). A similar tendency was noted for the right amygdala $\left[(x y z)=(261-20) ; z=2.61 ; p_{\text {uncorr }}=\right.$ 0.005 ; 98 voxels; Cohen's $d=1.19$ ].
To test the robustness of these findings, we reanalyzed amygdala $\mathrm{rCBF}$ data with the addition of a third placebo cohort of 12 subjects (one responder), taken from a similarly designed PET study (Furmark et al., 2005). Results confirmed a significantly greater bilateral amygdala $\mathrm{rCBF}$ diminution in placebo responders $(n=$ 11) compared with nonresponders ( $n=$ 26). This effect was significant both in the left $[(x y z)=(-12-3-13) ; z=3.10 ; p=$ $0.014 ; 80$ voxels; Cohen's $d=1.13$ ] and right $[(x y z)=(281-22) ; z=2.68 ; p=$ 0.044; 116 voxels; Cohen's $d=0.96$ ] amygdala. Because this third cohort had a shorter treatment period ( 6 weeks instead of 8 ), and gene data were not available, it was not included in the subsequent analyses.

\section{Effects of the serotonin} transporter polymorphism Thirteen subjects (54\%) carried one or two copies of the 5-HTTLPR s allele, whereas the remaining 11 were homozygous for the 1 allele (Table 1). Analyses of rCBF changes from pre- to post-placebo treatment showed a significantly greater rCBF-decrease in 11 relative to s carriers in the left amygdala [ $(x y z)(-28-7-15)$; $z=3.57 ; p=0.004 ; 197$ voxels; Cohen's $d=1.80$ ] (Fig. 4A).

\section{Effects of the tryptophan}

hydroxylase-2 polymorphism

Sixteen $(67 \%)$ subjects were homozygous for the TPH2 G allele, whereas 8 subjects were carriers of the T allele (Table 1). Analyses showed a significantly greater $\mathrm{rCBF}$ decrease for the GG relative to $\mathrm{T}$ carriers in the right amygdala $[(x y z)=(24-1-20) ; z=3.49 ; p=0.005$; 207 voxels; Cohen's $d=1.75]$ and a similar tendency for the left amygdala $\left[(x y z)=(-201-14) ; z=2.39 ; p_{\text {uncorr }}=0.009 ; 78\right.$ voxels; Cohen's $d=1.10$ ] (Fig. $4 B$ ). The effect of the TPH2 polymorphism on reduced amygdala $\mathrm{rCBF}$ with placebo treatment was not confounded by 5 -HTTLPR status and vice versa as the distribution of 11 and $s$ alleles was not different across the GG and T genotypes (Fisher's exact, $p=0.29$ ).

\section{Gene-gene effects}

Nine subjects (38\%) carried both the ll and GG genotypes (11/GG group), whereas the other 15 subjects were carriers of at least one of the $\mathrm{s}$ or $\mathrm{T}$ genotypes previously associated with enhanced amygdala reactivity (ll/T, $n=2$; s/GG, $n=7$; s/T, $n=6$ ). When using this grouping of genotypes in an analysis of the combined effects of the two polymorphisms, the 11/GG group exhibited a significantly greater placebo-related $\mathrm{rCBF}$ decrease in the left amygdala $\left[\left(\begin{array}{lll}x & y & z\end{array}\right)=(-26-7-15) ; z=3.36 ; p=0.008 ; 209\right.$ voxels; Cohen's $d=1.77]$ in comparison with subjects carrying at least one s or T allele (Fig. 4C).

\section{Pre-post differences within groups}

Within the groups mentioned above, significantly reduced amygdala rCBF from pretreatment to posttreatment was observed only in responders, ll homozygotes, and GG homozygotes (supple- 
Table 1. Descriptive characteristics of the participants that received placebo in two different clinical trials (cohorts I and II)

\begin{tabular}{|c|c|c|c|c|}
\hline Variable & & Cohort I $(n=13)$ & Cohort II $(n=12)$ & Total $(n=25)$ \\
\hline \multirow[t]{2}{*}{ Sex } & Male $[n(\%)]$ & $6(46.2)$ & $3(25.0)$ & $9(36.0)$ \\
\hline & Female $[n(\%)]$ & $7(53.8)$ & $9(75.0)$ & $16(64.0)$ \\
\hline \multirow[t]{2}{*}{ Age } & Mean (SD) & $37.8(9.1)$ & $33.1(12.1)$ & $35.6(10.7)$ \\
\hline & Range (min-max) & $24-49$ & $22-55$ & $22-55$ \\
\hline \multirow[t]{2}{*}{ Subtype } & Generalized $[n(\%)]$ & $9(69.2)$ & $9(75.0)$ & $18(72.0)$ \\
\hline & Nongeneralized $[n(\%)]$ & $4(30.8)$ & $3(25.0)$ & $7(28.0)$ \\
\hline \multirow[t]{2}{*}{ CGI response } & Responder $[n(\%)]$ & $5(38.5)$ & $5(41.7)$ & $10(40.0)$ \\
\hline & Nonresponder $[n(\%)]$ & $8(61.5)$ & $7(58.3)$ & $15(60.0)$ \\
\hline \multicolumn{5}{|l|}{$G_{\text {Genotype }}^{a}$} \\
\hline \multirow[t]{2}{*}{ 5-HTTLPR } & $\|[n(\%)]$ & $4(33.3)$ & $7(58.3)$ & $11(45.8)$ \\
\hline & $s[n(\%)]$ & $8(67.7)$ & $5(41.7)$ & $13(54.2)$ \\
\hline \multirow{2}{*}{$\mathrm{TPH} 2$} & $\mathrm{GG}[n(\%)]$ & $8(67.7)$ & $8(67.7)$ & $16(67.7)$ \\
\hline & $\mathrm{T}[n(\%)]$ & $4(33.3)$ & $4(33.3)$ & $8(33.3)$ \\
\hline \multirow[t]{2}{*}{ 5-HTTLPR/TPH2 } & II/GG $[n(\%)]$ & $4(33.3)$ & $5(41.7)$ & $9(37.5)$ \\
\hline & II/T, s/T, s/GG $[n(\%)]$ & $8(67.7)$ & $7(58.3)$ & $15(62.5)$ \\
\hline \multicolumn{5}{|c|}{ CGI responder by genotype } \\
\hline \multirow[t]{2}{*}{ 5-HTTLPR } & $\|[n(\%)]$ & $1(25.0)$ & $3(42.8)$ & $4(36.4)$ \\
\hline & $s[n(\%)]$ & $3(37.5)$ & $2(40.0)$ & $5(38.5)$ \\
\hline \multirow[t]{2}{*}{ TPH2 } & $\mathrm{GG}[n(\%)]$ & $3(37.5)$ & $5(62.5)$ & $8(50.0)$ \\
\hline & $\mathrm{T}[n(\%)]$ & $1(25.0)$ & $0(0)$ & $1(12.5)$ \\
\hline \multirow[t]{2}{*}{ 5-HTTLPR/TPH2 } & II/GG $[n(\%)]$ & $1(25.0)$ & $3(60.0)$ & $4(44.4)$ \\
\hline & II/T, s/T, s/GG $[n(\%)]$ & $3(37.5)$ & $2(28.6)$ & $5(33.3)$ \\
\hline
\end{tabular}

${ }^{a}$ Genotyping was performed on 24 of the 25 subjects. 5-HTTLPR, Serotonin transporter polymorphism (ll, long allele homozygotes; s, short allele carriers); TPH2, tryptophan hydroxylase-2 gene ( $\mathrm{G}$ allele homozygotes vs T allele carriers).

mental Table S1, available at www. jneurosci.org as supplemental material).

\section{Predictors of the clinical \\ placebo response}

In a logistic regression analysis, the TPH2 polymorphism emerged as the only significant variable that could reliably predict clinical placebo response (CGI-I) on day 56 (Table 2), homozygosity for the G allele being associated with better outcome. Eight of the nine placebo responders $(89 \%)$, for whom TPH2 gene data were available, were GG homozygotes. To avoid overfitting the regression model, we did
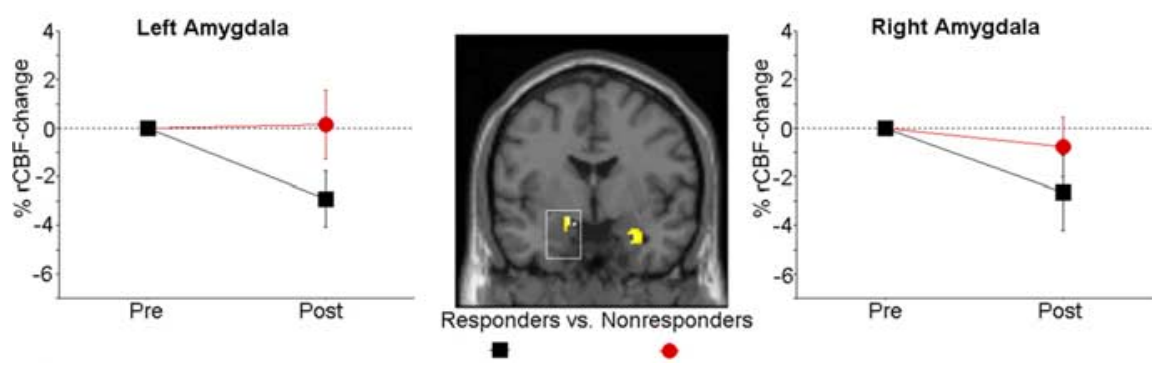

Figure 3. Placebo-induced alterations of rCBF during public speaking in the left and right amygdala. Placebo responders, compared with nonresponders, exhibited a significantly greater attenuation of rCBF in the left amygdala, and a subthreshold effect in the same direction was noted for the right amygdala. The mask threshold corresponds to $z>2$, and alterations that remained significant after correction for multiple comparisons are displayed within squares. the TPH2, 5-HTTLPR, left and right amygdala at baseline. The Hosmer and Lemeshow test indicated that the model adequately fitted the data $\left(\chi^{2}=9.40 ; \mathrm{df}=8 ; p=0.31\right)$. The model accounted for between 26.0 and $35.4 \%$ of the variance in CGI response (omnibus $\chi^{2}=7.21$; $\mathrm{df}=4 ; p=0.12$ ), and overall $70.8 \%$ of the predictions were accurate. The TPH 2 polymorphism remained a significant predictor $(p \leq 0.05)$ of the clinical placebo response after excluding all other predictor variables from the model except the right amygdala.

To test whether the influence of the TPH2 polymorphism on the clinical placebo response (CGI-I score) was dependent on its influence on the amygdala, path analysis was performed with the aim to find the best-fitting model from three possible candidates: (1) TPH2 $\rightarrow$ amygdala $\rightarrow$ CGI-I; (2) TPH2 $\rightarrow$ CGI-I $\rightarrow$ amygdala; (3) TPH2 $\rightarrow$ amygdala, TPH2 $\rightarrow$ CGI-I. The lowest BIC value, indicating the best fit (Raftery, 1993), was obtained for the model with paths from the TPH 2 to the amygdala and from the amygdala to CGI-I ( $p=$ 0.29 ) (Fig. 5). The TPH2 polymorphism explained 13\% of the variance in CGI-I at posttreatment and $33 \%$ of the variance in amygdala change scores, which dropped to $22 \%$ when the variance shared between the TPH 2 polymorphism and clinical improvement was partialed out. This suggests that the variance in amygdala activity explained by the TPH2 is not independent of the variance shared between the TPH2 and CGI-I, supporting that the genetic effect on placebo improvement is mediated by its effect on amygdala activity.

\section{PET data: opioid network}

At corrected $p$ levels, no differences in activity were found in placebo responders compared with nonresponders in four selected opioidrelevant ROIs (the anterior cingulate, orbitofrontal, and lateral prefrontal cortices, as well as the brainstem). At the uncorrected ( $p<$ $0.005)$ level, we noted higher placebo-induced activations in responders than nonresponders in the left prefrontal cortex $[(x y z)=$ $(-32626) ; z=2.70 ; 52$ voxels; Cohen's $d=1.24$; area 10], and conversely a relatively higher activation in the nonresponder group in the right orbitofrontal $[(x y z)=(1644-14) ; z=2.79 ; 419$ voxels; Cohen's $d=1.28$; area 11], right anterior cingulate $[(x y z)=(628$ $21) ; z=2.81 ; 400$ voxels; Cohen's $d=1.29$; area 24] and left prefrontal $[(x y z)=(-322336) ; z=2.71 ; 82$ voxels; Cohen's $d=1.24$; area 9] cortices. We did not find significant positive or negative covariations between $\mathrm{rCBF}$ in the amygdala and the selected regions.

\section{PET data: whole-brain analyses}

Exploratory analyses did not reveal significantly different treatment-induced patterns of change in responders versus non- 
A
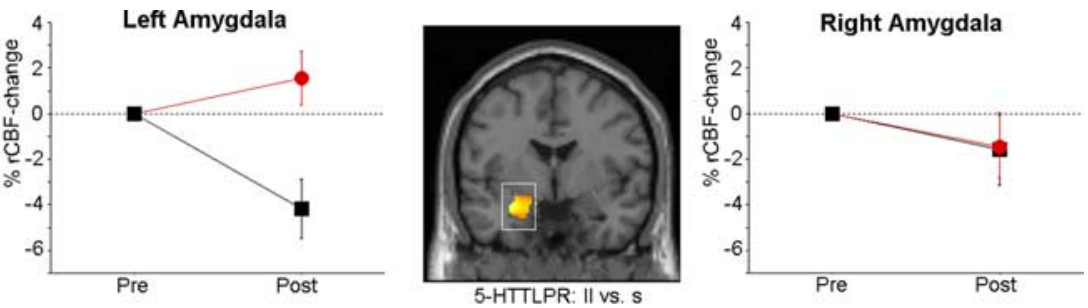

B
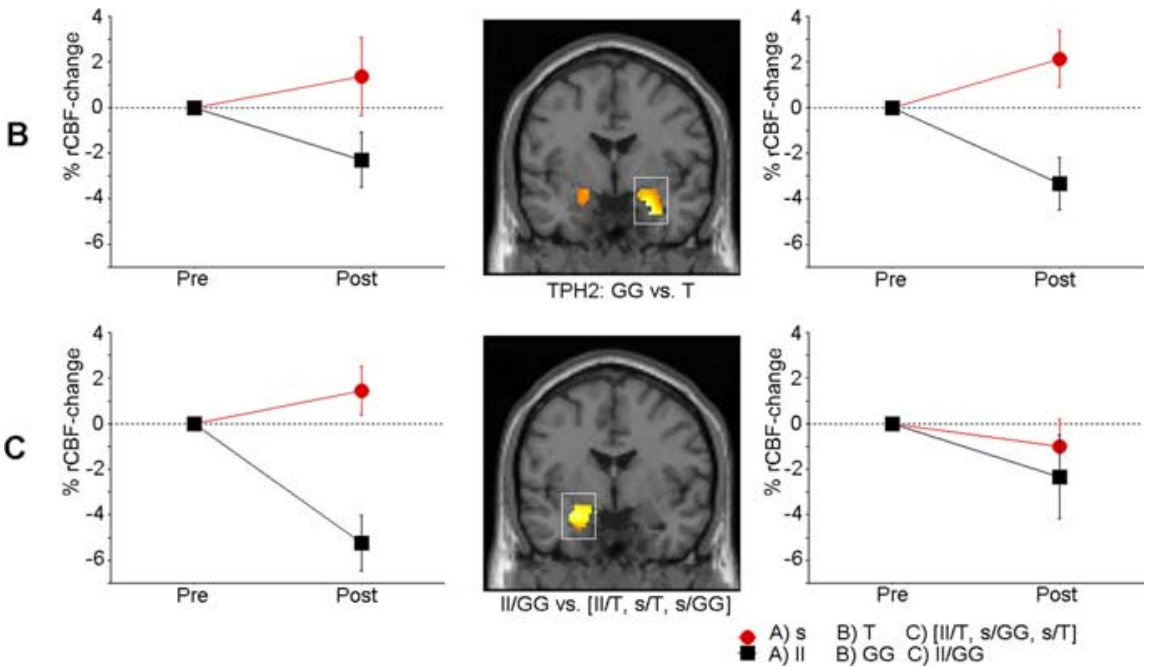

Figure 4. Placebo-induced alterations of $\mathrm{rCBF}$ during public speaking in the left and right amygdala in genetic subgroups. A significantly greater reduction of amygdala rCBF from pre- to post-placebo treatment was observed in the following: long allele (II) homozygotes compared with short (s) allele carriers of the serotonin transporter gene (5-HTTLPR) (A); G allele (GG) homozygotes compared with T allele carriers of the TPH2 G-703T polymorphism (B); and long and $\mathrm{G}$ allele homozygous patients (II/GG group) compared with patients carrying s and/or T alleles (II/T, s/GG, s/T group) (C). The mask threshold corresponds to $z>2$, and alterations that remained significant after correction for multiple comparisons are displayed within squares.

Table 2. Results from logistic regression analysis [i.e., coefficients (B), SE, Wald statistic, df, and probability values for predictor variables of the clinical placebo response at posttreatment]

\begin{tabular}{lrrrrr}
\hline Variable & $B$ & \multicolumn{1}{c}{ SE } & Wald & df & $p$ value \\
\hline TPH2 & 3.88 & 1.90 & 4.16 & 1 & 0.04 \\
5-HTTLPR & 1.44 & 1.33 & 1.18 & 1 & 0.28 \\
L Amygdala & 7.10 & 9.16 & 0.60 & 1 & 0.44 \\
R Amygdala & -18.24 & 11.38 & 2.57 & 1 & 0.11 \\
Constant & 12.44 & 14.31 & 0.76 & 1 & 0.39 \\
\hline
\end{tabular}

L, Left; R, right.

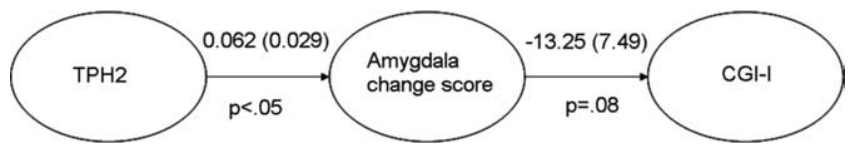

Figure 5. Path coefficients (SE) for the best fitting model that had paths from the TPH2 polymorphism to the amygdala and from the amygdala to the placebo response phenotype as assessed by the CGI-I.

responders. Significant within-group alterations outside the amygdala region were noted only in nonresponders, who had increased (pre $<$ post) rCBF in the right cerebellum $[(x y z)=(28$ $-42-23) ; z=4.73 ; p=0.038 ; 182$ voxels] and in a cluster encompassing the right primary motor and somatosensory cortices $[(x y z)=(38-2862) ; z=4.20 ; p<0.001 ; 1201$ voxels].

\section{Pretreatment differences}

There were no significant differences between placebo responders and nonresponders in sex distribution (Fisher's exact, $p>$
$0.22)$, age $(t=0.11$; $\mathrm{df}=23 ; p>0.91)$, or on the clinical outcome measures $(0.31<$ $t<1.36$; df $=23 ; p>0.19$ ) at baseline (day $0)$. Moreover, there were no significant differences between the genetic subgroups [ll vs s; GG vs T; ll/GG vs (ll/T, s/GG, s/T)] for any of these variables (all $p$ values $>$ 0.27). Similarly, whole-brain analyses did not reveal significant rCBF differences at baseline between responders and nonresponders, or between the genetic subgroups mentioned above, and in the amygdala volume the only significant effect was a higher baseline activity in 11 homozygotes relative to $\mathrm{s}$ carriers in the left hemisphere $[(x y z)=-16-5-15 ; z=$ $2.75 ; p=0.033 ; 51$ voxels; Cohen's $d=$ $1.30]$.

\section{Discussion}

The present study demonstrates that the magnitude of the placebo response, after prolonged treatment of patients diagnosed with SAD, is tied to attenuated amygdala excitability, which in turn is linked to serotonergic genetic variation. Stress-related activity in the amygdala was significantly more reduced in placebo responders than in nonresponders and only the patients who were homozygous for the 1 allele of the 5-HTTLPR and the G allele of the TPH2 G-703T polymorphism exhibited significantly attenuated amygdala responsiveness after placebo treatment, whereas carriers of the s and $\mathrm{T}$ alleles did not. Intriguingly, allelic variation in the TPH2 gene could accurately predict the clinical placebo response, and path analytic models supported that the genetic influence on symptom improvement with sustained placebo treatment is mediated by its influence on amygdala activity. Mediation analysis of this kind is an important advance in the field of imaging genetics.

The present results are consistent with the notion that placebo and active pharmacological agents act on the same, or at least overlapping, neural pathways (Mayberg et al., 2002; Petrovic et al., 2002, 2005; Benedetti et al., 2005; Faria et al., 2008). Pharmacological as well as cognitive-behavioral treatments of SAD have previously been shown to attenuate amygdala hyperactivity during aversive conditions (Furmark et al., 2002, 2005). Congruently, the current study found significantly greater reduction of stress-related amygdala activity in placebo responders compared with nonresponders and the robustness of this finding was further supported when merging data from a shorter but otherwise similarly designed clinical RCT (Furmark et al., 2005).

There are some reports of reduced amygdala responsiveness after successful therapy in other anxiety disorders (Goossens et al., 2007; Peres et al., 2007) as well as in depression (Sheline et al., 2001; Fu et al., 2004). Also in healthy volunteers, amygdala deactivation has been noticed after chronic administration of pharmacological agents with anxiety-reducing properties like serotonin reuptake inhibitors (Harmer et al., 2006; Arce et al., 2008) and benzodiazepines (Paulus et al., 2005; Arce et al., 2006). Acute drug exposure, however, may lead to potentiated amygdala reactivity (Bigos et al., 2008). Nonetheless, in a more long-term per- 
spective, lowered amygdala responsiveness may be important for anxiety alleviation, regardless of treatment modality.

The current results point to a modulatory role for serotonin in placebo-induced anxiety relief. The amygdala is densely innervated by serotonergic fibers (Bauman and Amaral, 2005), and imaging data support that amygdala responsiveness is influenced by synaptic serotonin (Fisher et al., 2006; Rhodes et al., 2007). As mentioned, studies of healthy volunteers have demonstrated that the serotonin-related 5-HTTLPR and TPH2 G-703T polymorphisms modulate the amygdala response to emotional stimuli. In this respect, the $\mathrm{s}$ and $\mathrm{T}$ variants appear to be "high-response" alleles, whereas the 1 and $G$ variants (i.e., 11 and/or GG homozygosity) are "low-response" alleles (Hariri et al., 2002; Brown et al. 2005; Canli et al., 2005; Hariri and Holmes, 2006). Congruently, the present data showed that the neural placebo effect, indexed by attenuated stressed-related activity in the amygdala after treatment, was more pronounced in patients who were 11 and/or GG homozygotes compared with carriers of the s and T alleles. Patients carrying both "low-response" alleles (i.e., the ll/GG group) exhibited a significantly greater placebo-related $\mathrm{rCBF}$ decrease in the left amygdala in comparison with subjects carrying at least one s or T allele. Notably, an additive effect of the s and T alleles on neural responding to emotional stimuli has been reported previously (Herrmann et al., 2007; Canli et al., 2008).

It could be speculated that dopaminergic or opioid processes are involved in the placebo response. PET studies have linked at least some types of placebo responses to reward expectancies and increased dopamine release (de la Fuente-Fernández et al., 2001; Scott et al., 2007), and other studies have tied placebo analgesic effects to activation of the endogenous opioid system (Petrovic et al., 2002; Zubieta et al., 2005) (but see Wager et al., 2007), which may be deficient in anxiety-disordered patients (Sher, 1998). Petrovic et al. (2005) examined the effect of experimental placebo on affective face processing in healthy volunteers who were led to expect differential emotional effects of either a benzodiazepine or a benzodiazepine receptor antagonist. The emotional placebo effect shared several key regions with those previously found in placebo analgesia, regions that have been interpreted to be parts of an opioid neuromodulatory system (Petrovic et al., 2002). Interestingly, and consistent with the present findings, the individual placebo response correlated with decreased activity in the amygdala (Petrovic et al., 2005). However, we could not demonstrate unequivocal evidence for involvement of the opioidrelevant brain areas previously implicated in imaging studies of placebo analgesia (Petrovic et al., 2002; Wager et al., 2004).

To the best of our knowledge, serotonin-related or other functional polymorphisms have not previously been linked to placebo response. One study noted inferior response in carriers of the 5-HTTLPR s allele during a placebo run-in period preceding serotonergic drug treatment (Rausch et al., 2002). The s allele has also been related to poorer response to antidepressants in clinical populations (Serretti et al., 2006) including SAD (M. B. Stein et al., 2006). In contrast, we could not verify predictive accuracy for the serotonin transporter polymorphism with regard to clinical improvement. However, TPH2 genetic variation was associated with the placebo response both neurally and behaviorally. It is noteworthy that the TPH2 GG homozygotes, who strongly dominated the responder group, exhibited bilateral rCBF decrement in the amygdala, and, in contrast to ll carriers, the statistical maximum was in the right hemisphere. However, it is unclear how hemispheric asymmetries in the amygdala relate to treatment outcome because left and right as well as bilateral rCBF reduc- tions have previously been associated with reduced anxiety in SAD patients (Furmark et al., 2002, 2005).

The current data imply that placebo-induced attenuation of amygdala activity can be regarded as an endophenotype linked to the clinical response, which in turn is under the influence of the $\mathrm{TPH} 2$ polymorphism. In a similar way, enhanced baseline amygdala responsivity, characteristic for carriers of the 5-HTTLPR s and TPH2 T alleles (Hariri et al., 2002; Brown et al. 2005; Canli et al., 2005; Hariri and Holmes, 2006), may underlie refractoriness to placebo treatment. Consistent with this notion, a recent study of generalized anxiety disorder reported that lesser pretreatment reactivity in the amygdala predicted greater decreases in anxiety after 8 weeks of treatment with venlafaxine (Whalen et al., 2008). Because our neuroimaging paradigm did not include a neutral control task, we could not properly evaluate pretreatment amygdala reactivity. Also because of this limitation, we could not evaluate whether the attenuated amygdala activity with placebo reflects reduced reactivity to the stressful task or a lowered trait-like activity level. However, in a previous study, we found some support for the notion that amygdala deactivation is specifically related to state anxiety reduction (Furmark et al., 2005).

The present study adds to the existing imaging-placebo literature by evaluating genetic influences on the placebo response, by statistically comparing placebo responders and nonresponders, by using a typical double-blind pharmaceutical RCT model and by assessing prolonged rather than acute placebo treatment. However, our study was not powered to detect associations with modest effect sizes, for example, potential small-size effects of the 5-HTTLPR on the clinical placebo response. Moreover, to avoid mass comparisons, we focused on only two polymorphisms with known modulatory effects on the amygdala. There are, however, several relevant serotonin-related and other genes that could be addressed in future work, such as the serotonin-1A-receptor gene $\left(5-\mathrm{HT}_{1 \mathrm{~A}}-1019 \mathrm{C} / \mathrm{G}\right)$ (Dannlowski et al., 2007) and the catecholO-methyltransferase gene (COMT Val158Met) (Smolka et al., 2005). Functional connectivity analyses could be used in future studies to examine how genes affect not only the amygdala response to placebo but also pathways in a larger affective processing network. It should also be noted that there are probably many types of placebo responses with varying biological underpinnings (Benedetti et al., 2005), and the current findings on placebo may or may not be relevant for other settings, populations, and target behaviors.

Although the present report demonstrates that serotoninrelated polymorphisms influence amygdala activity and the propensity to respond to placebo in patients with SAD, additional work is necessary to elucidate the generalizability of the findings. We predict, however, that studies combining neuroimaging and molecular genetic techniques will reveal similar genetic influences also on other placebo-responsive conditions, which could have important consequences for the planning and conduct of RCTs. Meanwhile, the data presented here show the first evidence of a link between serotonin-related genotypes, activity in a brain region crucial for emotional processing, and the placebo response phenotype in individuals treated for social anxiety.

\section{References}

American Psychiatric Association (1994) Diagnostic and statistical manual of mental disorders, Ed 4. Washington, DC: American Psychiatric Press.

Arce E, Miller DA, Feinstein JS, Stein MB, Paulus MP (2006) Lorazepam dose-dependently decreases risk-taking related activation in limbic areas. Psychopharmacology 189:105-116.

Arce E, Simmons AN, Lovero KL, Stein MB, Paulus MP (2008) Escitalo- 
pram effects on insula and amygdala BOLD activation during emotional processing. Psychopharmacology 196:661-672.

Bauman MD, Amaral DG (2005) The distribution of serotonergic fibers in the macaque monkey amygdala: an immunohistochemical study using antisera to 5-hydroxytryptamine. Neuroscience 36:193-203.

Beecher HK (1955) The powerful placebo. JAMA 159:1602-1606.

Benedetti F, Mayberg HS, Wager TD, Stohler CS, Zubieta JK (2005) Neurobiological mechanisms of the placebo effect. J Neurosci 25:10390-10402.

Bigos KL, Pollock BG, Aizenstein HJ, Fisher PM, Bies RR, Hariri AR (2008) Acute 5-HT reuptake blockade potentiates human amygdala reactivity. Neuropsychopharmacology 33:3221-3225.

Brown SM, Peet E, Manuck SB, Williamson DE, Dahl RE, Ferrell RE, Hariri AR (2005) A regulatory variant of the human tryptophan hydroxylase-2 gene biases amygdala reactivity. Mol Psychiatry 10:884-888.

Canli T, Congdon E, Gutknecht L, Constable RT, Lesch KP (2005) Amygdala responsiveness is modulated by tryptophan hydroxylase-2 gene variation. J Neural Transm 112:1479-1485.

Canli T, Congdon E, Todd Constable R, Lesch KP (2008) Additive effects of serotonin transporter and tryptophan hydroxylase-2 gene variation on neural correlates of affective processing. Biol Psychol 79:118-125.

Dannlowski U, Ohrmann P, Bauer J, Kugel H, Baune BT, Hohoff C, Kersting A, Arolt V, Heindel W, Deckert J, Suslow T (2007) Serotonergic genes modulate amygdala activity in major depression. Genes Brain Behav 6:672-676.

de la Fuente-Fernández R, Ruth TJ, Sossi V, Schulzer M, Calne DB, Stoessl AJ (2001) Expectation and dopamine release: mechanism of the placebo effect in Parkinson's disease. Science 293:1164-1166.

Faria V, Fredrikson M, Furmark T (2008) Imaging the placebo response: a neurofunctional review. Eur Neuropsychopharmacol 18:473-485.

First MB, Gibbon M, Spitzer RL, Williams JBW (1998) SCID-I: interview protocol (in Swedish). Stockholm: Pilgrim.

Fisher PM, Meltzer CC, Ziolko SK, Price JC, Moses-Kolko EL, Berga SL, Hariri AR (2006) Capacity for 5-HT1A-mediated autoregulation predicts amygdala reactivity. Nat Neurosci 9:1362-1363.

Friston KJ, Holmes AP, Worsley KJ, Poline JB, Frith CD, Frackowiak RSJ (1995) Statistical parametric maps in functional imaging: a general linear approach. Hum Brain Mapp 2:189-210.

Fu CH, Williams SC, Cleare AJ, Brammer MJ, Walsh ND, Kim J, Andrew CM, Pich EM, Williams PM, Reed LJ, Mitterschiffthaler MT, Suckling J, Bullmore ET (2004) Attenuation of the neural response to sad faces in major depression by antidepressant treatment: a prospective, event-related functional magnetic resonance imaging study. Arch Gen Psychiatry 61:877-889.

Furmark T, Tillfors M, Marteinsdottir I, Fischer H, Pissiota A, Långström B, Fredrikson M (2002) Common changes in cerebral blood flow in patients with social phobia treated with citalopram or cognitive-behavioral therapy. Arch Gen Psychiatry 59:425-433.

Furmark T, Tillfors M, Garpenstrand H, Marteinsdottir I, Långström B, Oreland L, Fredrikson M (2004) Serotonin transporter polymorphism related to amygdala excitability and symptom severity in patients with social phobia. Neurosci Lett 362:189-192.

Furmark T, Appel L, Michelgård A, Wahlstedt K, Ahs F, Zancan S, Jacobsson E, Flyckt K, Grohp M, Bergström M, Pich EM, Nilsson LG, Bani M, Långström B, Fredrikson M (2005) Cerebral blood flow changes after treatment of social phobia with the neurokinin-1 antagonist GR205171, citalopram, or placebo. Biol Psychiatry 58:132-142.

Gelernter J, Kranzler H, Cubells JF (1997) Serotonin transporter protein (SLC6A4) allele and haplotype frequencies and linkage disequilibria in African- and European-American and Japanese populations and in alcohol-dependent subjects. Hum Genet 101:243-246.

Goossens L, Sunaert S, Peeters R, Griez EJ, Schruers KR (2007) Amygdala hyperfunction in phobic fear normalizes after exposure. Biol Psychiatry 62:1119-1125.

Hariri AR, Holmes A (2006) Genetics of emotional regulation: the role of the serotonin transporter in neural function. Trends Cogn Sci 10:182-191.

Hariri AR, Mattay VS, Tessitore A, Kolachana B, Fera F, Goldman D, Egan MF, Weinberger DR (2002) Serotonin transporter genetic variation and the response of the human amygdala. Science 297:400-403.

Harmer CJ, Mackay CE, Reid CB, Cowen PJ, Goodwin GM (2006) Antidepressant drug treatment modifies the neural processing of nonconscious threat cues. Biol Psychiatry 59:816-820.
Herrmann MJ, Huter T, Müller F, Mühlberger A, Pauli P, Reif A, Renner T, Canli T, Fallgatter AJ, Lesch KP (2007) Additive effects of serotonin transporter and tryptophan hydroxylase-2 gene variation on emotional processing. Cereb Cortex 17:1160-1163.

Lancaster JL, Woldorff MG, Parsons LM, Liotti M, Freitas CS, Rainey L, Kochunov PV, Nickerson D, Mikiten SA, Fox PT (2000) Automated Talairach atlas labels for functional brain mapping. Hum Brain Mapp 10:120-131.

Liebowitz MR (1987) Social phobia. Mod Probl Pharmacopsychiatry 22:141-173.

Lorberbaum JP, Kose S, Johnson MR, Arana GW, Sullivan LK, Hamner MB, Ballenger JC, Lydiard RB, Brodrick PS, Bohning DE, George MS (2004) Neural correlates of speech anticipatory anxiety in generalized social phobia. Neuroreport 15:2701-2705.

Mai JK, Assheuer J, Paxinos G (2004) Atlas of the human brain. San Diego: Elsevier Academic.

Maldjian JA, Laurienti PJ, Kraft RA, Burdette JH (2003) An automated method for neuroanatomic and cytoarchitectonic atlas-based interrogation of fMRI data sets. Neuroimage 19:1233-1239.

Mattick RP, Clarke JC (1998) Development and validation of measures of social phobia scrutiny fear and social interaction anxiety. Behav Res Ther 36:455-470.

Mayberg H, Silva JA, Brannan SK, Tekell JL, Mahurin RK, McGinnis S, Jerabek PA (2002) The functional neuroanatomy of the placebo effect. Am J Psychiatry 159:728-737.

Muthen LK, Muthen BO (2008) Mplus user's guide, Ed 5. Los Angeles: Muthen and Muthen.

Paulus MP, Feinstein JS, Castillo G, Simmons AN, Stein MB (2005) Dose-dependent decrease of activation in bilateral amygdala and insula by lorazepam during emotion processing. Arch Gen Psychiatry 62:282-288.

Peres JF, Newberg AB, Mercante JP, Simão M, Albuquerque VE, Peres MJ, Nasello AG (2007) Cerebral blood flow changes during retrieval of traumatic memories before and after psychotherapy: a SPECT study. Psychol Med 37:1481-1491.

Petrovic P, Kalso E, Petersson KM, Ingvar M (2002) Placebo and opioid analgesia: imaging a shared neuronal network. Science 295:1737-1740.

Petrovic P, Dietrich T, Fransson P, Anderson J, Carlsson K, Ingvar M (2005) Placebo in emotional processing: induced expectations of anxiety relief activate a generalized modulatory network. Neuron 46:957-969.

Phan KL, Fitzgerald DA, Nathan PJ, Tancer ME (2006) Association between amygdala hyperactivity to harsh faces and severity of social anxiety in generalized social phobia. Biol Psychiatry 59:424-429.

Raftery AE (1993) Bayesian model selection in structural equation models. In: Testing structural equation models (Bollen KA, Long JS, eds), pp 163-180. Newbury Park, CA: Sage Publications.

Rausch JL, Johnson ME, Fei YJ, Li JQ, Shendarkar N, Hobby HM, Ganapathy V, Leibach FH (2002) Initial conditions of serotonin transporter kinetics and genotype: influence on SSRI treatment trial outcome. Biol Psychiatry 51:723-732.

Rhodes RA, Murthy NV, Dresne MA, Selvaraj S, Stavrakakis N, Babar S, Cowen PJ, Grasby PM (2007) Human 5-HT transporter availability predicts amygdala reactivity in vivo. J Neurosci 27:9233-9237.

Scott DJ, Stohler CS, Egnatuk CM, Wang H, Koeppe RA, Zubieta JK (2007) Individual differences in reward responding explain placebo-induced expectations and effects. Neuron 55:325-336.

Serretti A, Calati R, Mandelli L, De Ronchi D (2006) Serotonin transporter gene variants and behavior: a comprehensive review. Curr Drug Targets 7:1659-1669.

Sheehan DV, Lecrubier Y, Sheehan KH, Amorim P, Janavs J, Weiller E, Hergueta T, Baker R, Dunbar GC (1998) The Mini-International Neuropsychiatric Interview (M.I.N.I.): the development and validation of a structured diagnostic psychiatric interview for DSM-IV and ICD-10. J Clin Psychiatry 59 [Suppl 20]:22-33.

Sheline YI, Barch DM, Donnelly JM, Ollinger JM, Snyder AZ, Mintun MA (2001) Increased amygdala response to masked emotional faces in depressed subjects resolves with antidepressant treatment: an fMRI study. Biol Psychiatry 50:651-658.

Sher L (1998) The role of the endogenous opioid system in the pathogenesis of anxiety disorders. Med Hypotheses 50:473-474.

Smolka MN, Schumann G, Wrase J, Grüsser SM, Flor H, Mann K, Braus DF, Goldman D, Büchel C, Heinz A (2005) Catechol-O-methyltransferase 
val158met genotype affects processing of emotional stimuli in the amygdala and prefrontal cortex. J Neurosci 25:836-842.

Stein DJ, Baldwin DS, Dolberg OT, Despiegel N, Bandelow B (2006) Which factors predict placebo response in anxiety disorders and major depression? An analysis of placebo-controlled studies of escitalopram. J Clin Psychiatry 67:1741-1746.

Stein MB, Goldin PR, Sareen J, Zorrilla LT, Brown GG (2002) Increased amygdala activation to angry and contemptuous faces in generalized social phobia. Arch Gen Psychiatry 59:1027-1034.

Stein MB, Seedat S, Gelernter J (2006) Serotonin transporter gene promoter polymorphism predicts SSRI response in generalized social anxiety disorder. Psychopharmacology 187:68-72.

Talairach J, Tournoux P (1988) Co-planar stereotaxic atlas of the human brain. Stuttgart, Germany: Georg Thieme Verlag.

Tillfors M, Furmark T, Marteinsdottir I, Fischer H, Pissiota A, Långström B, Fredrikson M (2001) Cerebral blood flow in subjects with social phobia during stressful speaking tasks: a PET-study. Am J Psychiatry 158:1220-1226.
Tillfors M, Furmark T, Marteinsdottir I, Fredrikson M (2002) Cerebral blood flow during anticipation of public speaking in social phobia: a PET study. Biol Psychiatry 52:1113-1119.

Wager TD, Rilling JK, Smith EE, Sokolik A, Casey KL, Davidson RJ, Kosslyn SM, Rose RM, Cohen JD (2004) Placebo induced changes in fMRI in the anticipation and experience of pain. Science 303:1162-1167.

Wager TD, Scott DJ, Zubieta JK (2007) Placebo effects on human $\mu$-opioid activity during pain. Proc Natl Acad Sci U S A 104:11056-11061.

Whalen PJ, Johnstone T, Somerville LH, Nitschke JB, Polis S, Alexander AL, Davidson RJ, Kalin NH (2008) A functional magnetic resonance imaging predictor of treatment response to venlafaxine in generalized anxiety disorder. Biol Psychiatry 63:858-863.

Zaider TI, Heimberg RG, Fresco DM, Schneier FR, Liebowitz MR (2003) Evaluation of the clinical global impression scale among individuals with social anxiety disorder. Psychol Med 33:611-622.

Zubieta JK, Bueller JA, Jackson LR, Scott DJ, Xu Y, Koeppe RA, Nichols TE, Stohler CS (2005) Placebo effects mediated by endogenous opioid activity on $\mu$-opioid receptors. J Neurosci 25:7754-7762. 